\title{
Assessing the Liverpool Respiratory Symptom Questionnaire in children with cystic fibrosis
}

\author{
R. Trinick*,\#, K.W. Southern*,\#, and P.S. McNamara*,\#,
}

ABSTRACT: Monitoring respiratory status in cystic fibrosis (CF) is challenging, particularly in young children. We aimed to test whether the Liverpool Respiratory Symptom Questionnaire (LRSQ) could distinguish well, pre-school and older children with and without CF, whether it could distinguish well and unwell children with CF and, finally, whether LRSQ scores in older children with CF correlated with established measures of disease severity.

20 stable pre-school children with CF had significantly higher total LRSQ scores than 51 preschool controls, and higher scores in two out of eight domains. Similarly, 21 stable 6- to 12-yr-old children with CF had higher total scores than 97 6- to 12-yr-old controls, and higher scores in seven out of eight domains.

In older children with CF, LRSQ scores correlated negatively with Shwachman score and forced expiratory volume in $1 \mathrm{~s}(\mathrm{r}=-0.58, \mathrm{p}<0.001, \mathrm{n}=31$; and $\mathrm{r}=-0.46, \mathrm{p}<0.010, \mathrm{n}=34$, respectively). Within the CF group, patients who cultured Pseudomonas aeruginosa, who used more "back-up" antibiotics or whose school attendance was lower also had higher LRSQ scores.

The LRSQ differentiates well children from those with CF in both pre-school and the 6- to 12-yrold age group, even at a point of stability. It also differentiates stable from unwell children with $\mathrm{CF}$, and scores correlate with other measures of respiratory disease, highlighting its potential as a clinical monitoring tool in paediatric CF.

KEYWORDS: Cystic fibrosis, monitoring, paediatrics, patient-reported outcome measure, symptom score

$\mathbf{R}$ ecent improvements in cystic fibrosis (CF) survival have been attributed to prevention and timely, active treatment of chest disease. In an increasingly healthy population, measures to monitor respiratory status, including spirometry and chest radiograph scores [1], lack the required sensitivity to objectively detect deterioration in condition. Novel noninvasive measures, applicable to standard clinical practice, are needed to identify potential windows for early intervention $[2,3]$.

Recently, there has been considerable interest in patient-reported outcome (PRO) measures, with the National Institutes of Health aiming to develop a large test-bank of PRO measures useful in the clinical assessment of chronic disease $[4,5]$. The two main types of PRO are health-related quality of life questionnaires and symptom scores. In paediatric $\mathrm{CF}$, respiratory symptom score development has been limited, particularly in pre-school children, for whom assessment is difficult and reliant on parental reports or direct behavioural observations. To date, there is no CFspecific validated tool for this age group. Those tools that have been validated in children focus on the recognition of acute pulmonary exacerbations or short-term trial outcomes, rather than a longer term reflection of parent-reported respiratory condition. The Cystic Fibrosis Questionnaire Revised (CFQ-R) respiratory symptom scale has been well validated in the 6- to 13-yr-old age group, but is focussed on a 2-week recall period and has mainly been used in clinical trial outcomes rather than clinical monitoring [6-10].

We have previously validated a respiratory symptom questionnaire in non-CF pre-school children, one of few studies intending to attempt respiratory symptom score validation in this age group [11, 12]. The Liverpool Respiratory Symptom Questionnaire (LRSQ), which carefully documents respiratory symptoms across multiple domains, has proven to be sensitive at detecting respiratory disease in this young population. It has shown strong divergent construct validity,

\section{AFFILIATIONS}

*Dept of Women's and Children's Health, Institute of Translational Medicine, University of Liverpool, and

${ }^{\#}$ Respiratory Unit, Alder Hey Children's Hospital NHS Foundation Trust, Liverpool, UK. "These authors are joint senior authors.

CORRESPONDENCE P.S. McNamara Senior Lecturer in Child Health, Institute of Child Health, University of Liverpool Alder Hey Children's Hospital Eaton Road

Liverpool L12 2AP UK E-mail: mcnamp@liverpool.ac.uk

Received:

April 262011

Accepted after revision:

Aug 172011

First published online:

Sept 012011 
good internal consistency and short-term reliability of questions, and, importantly, is practical and acceptable for parents. The LRSQ is designed to detect symptoms over 3 months. This longer time period lessens the effect of acute changes in clinical condition, thus making this score more relevant to both healthcare professionals and parents when tracked over time.

In this study, we aimed to assess whether the LRSQ could distinguish between healthy children and children with stable $\mathrm{CF}$ in the pre-school age group. We also aimed to assess whether it could further distinguish between stable and unwell children within the CF group. In view of the known limitations of current clinical measures of respiratory status in pre-school children, we included a school age group (aged 6-12 yrs). This would enable us to assess correlations of LRSQ score with other objective measures of disease status, such as the Shwachman score, Northern chest X-ray (NCXR) scores and spirometry, giving an indication of the scale's sensitivity to disease status within the paediatric CF population.

\section{MATERIAL AND METHODS Study subjects}

Parents of children with and without CF completed the LRSQ following written consent for inclusion in the study.

All patients were recruited at routine clinic appointments and were included if they had CF and were aged 0-12 yrs. All were prescribed antibiotic prophylaxis. There is no agreed definition for "stability" in CF and, therefore, this definition was agreed locally amongst a group of respiratory paediatricians. Children were deemed "stable" if they had needed no more than one course of back-up oral antibiotics in the preceding 3 months and this course had not been within the previous 2 weeks.

Healthy controls without respiratory disease were either recruited from local nurseries and schools or were children of members of staff or friends of patients admitted to Alder Hey Children's Hospital (Liverpool, UK). Exclusion criteria included children with chronic illnesses or those needing regular medications. The Liverpool Local Research Ethical Committee approved the study $(02 / 07 / 108 / \mathrm{A}(\mathrm{C}))$.

\section{Study design}

This was a questionnaire based, cross-sectional study.

\section{LRSQ and outcome measures}

The LRSQ consists of eight domains, each containing between three and five items. The first six domains assess respiratory symptoms and the remaining two assess the impact of symptoms on the child and family (table 1). Parents were asked to consider symptoms over the last 3 months, with questions being scored on a five-point Likert scale from "not at all" (score 0 ) to "every day" (score 4). A score for each domain and for the complete questionnaire was calculated (maximum score 128).

Demographic information including age, sex and number of smokers in the household were recorded. Children with CF had their recent NCXR score, Shwachman score, spirometry results, Pseudomonas aeruginosa carriage, antibiotic usage and school attendance (over the last 3 months) recorded. A single radiologist scored all chest radiographs [1]. The Shwachman score comprises four domains reflecting respiratory symptoms, activity, nutritional status and chest radiograph appearances [13]. P. aeruginosa carriage was recorded in two ways. First, on the clinic specimen at the time of the study and, secondly, on a four-point scale of carriage according to the Leeds criteria [14]. Clinic spirometry results were recorded at the time of questionnaire completion and children were classified by percentage predicted forced expiratory volume in $1 \mathrm{~s}$ (FEV1) according to established cut-off points for "mild" (FEV1 >70\%), "moderate" (FEV1 40-69\%) or "severe" $(<40 \%)$ disease [15].

\section{Statistical analysis}

SPSS version 18.0 (SPSS Inc., Chicago, IL, USA) was used. Data are expressed as median (interquartile range). The MannWhitney U-test was used for comparison of ages between the $\mathrm{CF}$ and healthy control groups. The Chi-squared test was used for comparison of sex and number of smokers per household between groups. Following log transformation of LRSQ scores, multiple linear regression analysis was performed to compare domain and total scores between healthy controls and stable children with $\mathrm{CF}$, taking account of potential confounders between groups such as differences in number of smokers per household. A Bonferroni correction was used to minimise the risk of type 1 error. Spearman's rank correlation was used to examine the correlation of total LRSQ scores with NCXR scores, the Shwachman score, and FEV1 and forced vital capacity (FVC). The Kruskal-Wallis H-test was used to compare total LRSQ scores across multiple categorical groups.

Cronbach's $\alpha$ coefficients were calculated to assess internal consistency of items within individual questionnaire domains, indicating the extent to which they measured the same construct. Coefficients of $r=0.70$ were considered acceptable [16].

Ceiling and floor effects (the percentage of children scoring at scale extremes) within LRSQ total scores were studied. Ceiling or floor effects of $>20 \%$ are considered significant $[17,18]$.

\section{TABLE 1 Details of the components of the Liverpool Respiratory Symptom Questionnaire}

Questionnaire domain

Daytime symptoms, symptoms with colds, interval symptoms (between colds), symptoms with activity

Night-time symptoms

Other symptoms

Effects on child

Effects on family
Symptoms assessed

Cough, wheeze, shortness of breath, "rattly" chest

Cough, wheeze, shortness of breath, "rattly" chest, snoring Noisy breathing not from chest, noisy breathing from throat, fast breathing Feeding, activity levels, sleep disturbance, fatigue Family activities, adjustment to family life, disturbed sleep, worry/anxiety 


\begin{tabular}{|c|c|c|c|c|}
\hline TABLE 2 & $\begin{array}{l}\text { l and doma } \\
\text { control gro }\end{array}$ & $\begin{array}{l}\text { scores } \\
\text { ps }\end{array}$ & or cystic fib & sis (CF) \\
\hline \multirow{2}{*}{$\begin{array}{l}\text { Questionnaire } \\
\text { domain }\end{array}$} & \multicolumn{2}{|c|}{$\leqslant 5$ yrs } & \multicolumn{2}{|c|}{$6-12$ yrs } \\
\hline & CF patients & Controls & CF patients & Controls \\
\hline Subjects n & 20 & 51 & 21 & 97 \\
\hline $\begin{array}{l}\text { Daytime symptom } \\
\text { score }\end{array}$ & $1.5(1-3)$ & $1(0-2)$ & $3(1-5)^{\star \star}$ & $0(0-1)$ \\
\hline $\begin{array}{l}\text { Night-time } \\
\text { symptom score }\end{array}$ & $2(1-3.75)$ & $1(0-2)$ & $2(1-3.5)^{\star \star}$ & $1(0-2)$ \\
\hline $\begin{array}{l}\text { Cold symptom } \\
\text { score }\end{array}$ & $3(1-8)$ & $1(1-3)$ & $3(1-5)$ & $1(0-2)$ \\
\hline $\begin{array}{l}\text { Interval symptom } \\
\text { score }\end{array}$ & $0(0-1.25)$ & $0(0-1)$ & $1(0-3)^{\star \star}$ & $0(0-0)$ \\
\hline $\begin{array}{l}\text { Activity symptom } \\
\text { score }\end{array}$ & $0.5(0-2)$ & $0(0-1)$ & $1(0-6)^{* *}$ & $0(0-0)$ \\
\hline $\begin{array}{l}\text { Other symptom } \\
\text { score }\end{array}$ & $0(0-2.75)^{\star}$ & $0(0-0)$ & $0(0-3)^{\star \star}$ & $0(0-0)$ \\
\hline $\begin{array}{l}\text { Effect on child } \\
\text { score }\end{array}$ & $0.5(0-2.75)$ & $0(0-1)$ & $1(0-3)^{\star \star}$ & $0(0-0)$ \\
\hline $\begin{array}{l}\text { Effect on family } \\
\text { score }\end{array}$ & $\begin{array}{l}2(0.25- \\
5.75)^{\star \star}\end{array}$ & $0(0-2)$ & $3(0.5-4.5)^{\star *}$ & $0(0-0)$ \\
\hline Total LRSQ score & $11.5(8-20)^{*}$ & $5(1-11)$ & $14(5-33)^{* *}$ & $3(0-6)$ \\
\hline
\end{tabular}

Data are presented as median (interquartile range), unless otherwise stated LRSQ: Liverpool Respiratory Symptom Questionnaire. *: $p<0.05$; **: $p<0.01$.

\section{RESULTS}

\section{Demographics and clinical details}

64 children with CF were recruited. One questionnaire was incomplete and therefore excluded, leaving 63 children in the CF group ( 31 aged $\leqslant 5$ yrs). 41 out of 63 children with CF (20 aged $\leqslant 5$ yrs) were considered to be "stable" at the time of questionnaire completion. 148 healthy controls were included (51 aged $\leqslant 5 \mathrm{yrs}$ ). The median age for both controls and children with CF in the $\leqslant 5$ yr-old age group was 3 yrs (range 3 months -5 yrs). The median age in the $>5$-yr-old age group

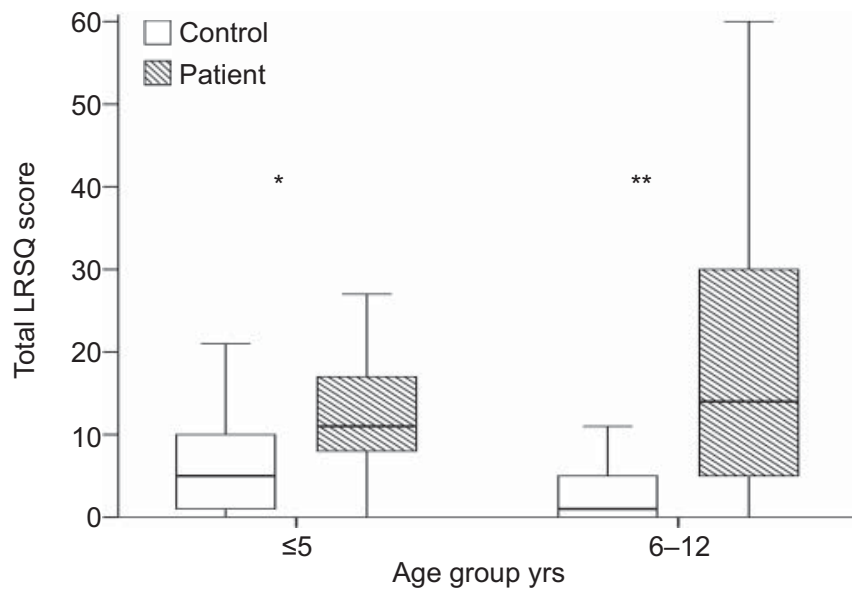

FIGURE 1. Total Liverpool Respiratory Symptom Questionnaire (LRSQ) scores for age groups $\leqslant 5$ and $6-12 \mathrm{yrs}$. Data are presented as median and interquartile range. ${ }^{*}: p<0.05 ; * *: p<0.01$ was 10 (range 6-12 yrs) for control children and 9 (range 612 yrs) for CF patients $(p=0.095)$. There was no significant difference in sex between the CF and control group (56\% and $46 \%$ male, respectively; $\mathrm{p}=0.259)$. There were significantly more smoking households in the $\mathrm{CF}$ group $(48 \%$ of $\mathrm{CF}$ households versus $21 \%$ in the control group; $\mathrm{p}<0.001)$.

Within the whole CF group ( $\mathrm{n}=63), 20 \%$ cultured $P$. aeruginosa persistently from sputum or cough swab at the time of LRSQ completion $(n=5$ in the $\leqslant 5$ yr group and $n=8$ in the $>5 \mathrm{yr}$ group). All children with $\mathrm{CF}$ aged $>5$ yrs $(n=32)$ completed spirometry. We classified FEV1 results according to established cut-offs for "mild" (FEV1 >70\%), "moderate" (FEV1 40-69\%) or "severe" $(<40 \%)$ disease. $19(58 \%)$ patients were within the "mild" category, and 13 patients were within the "moderate" category. No children were classified "severe" [15].

\section{Discriminant validity: $\leqslant 5$-yr-old age group}

Pre-school, "stable" children with CF $(n=20)$ scored significantly higher in total LRSQ score (median 11.5 (interquartile range $8-20)$ versus $5(1-11) ; \mathrm{p}<0.020)$ and two out of eight domain scores ("effects on family", p $<0.010$; and "Other symptoms", $\mathrm{p}<0.050$ ) than healthy controls (table 2, fig. 1 ). The difference in total score persisted even if only the eight children with CF entirely free of supplementary antibiotics for the study period were included in the analysis (10.5 (8.3-16.3) versus 5 (1-11); $\mathrm{p}<0.05)$. Examination of cross-sectional data for different age categories ( $<2$ yrs, $2-4$ yrs and $4-6$ yrs) showed that mean LRSQ scores in pre-school children with CF increased with age, and that the difference between CF and controls became more pronounced in older pre-school children (fig. 2).

No correlation between the LRSQ and NCXR scores or Shwachman score was seen. On assessing the entire group (stable and unstable, $\mathrm{n}=31$ ), a trend was seen in LRSQ scores dependent on the number of courses of antibiotics needed during the study period (fig. 3). Despite small study numbers, there was a significant difference in scores between those not needing supplementary antibiotics and those needing two courses $(\mathrm{p}<0.05)$.

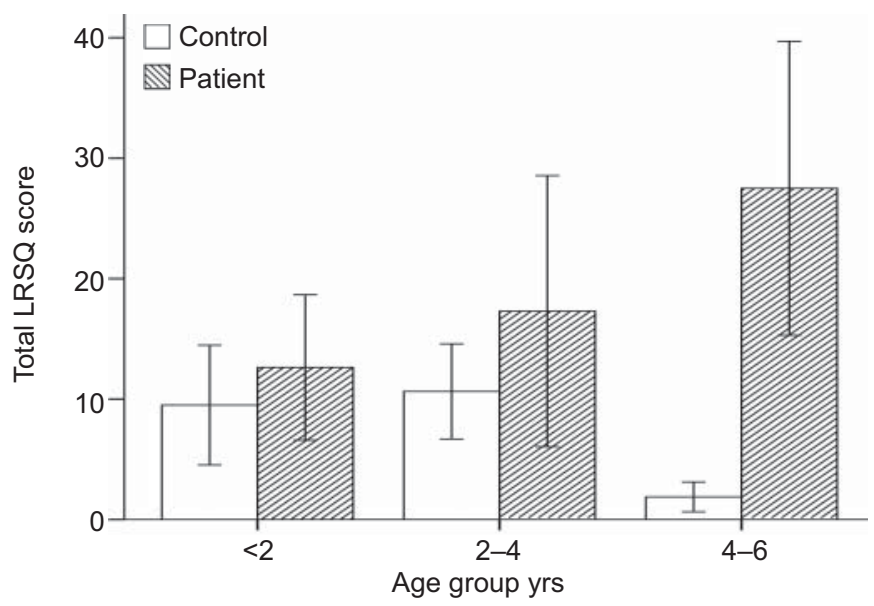

FIGURE 2. Total Liverpool Respiratory Symptom Questionnaire (LRSQ) scores for age groups $<2,2-4$ and $4-6$ yrs. Data are presented as mean \pm SE. 


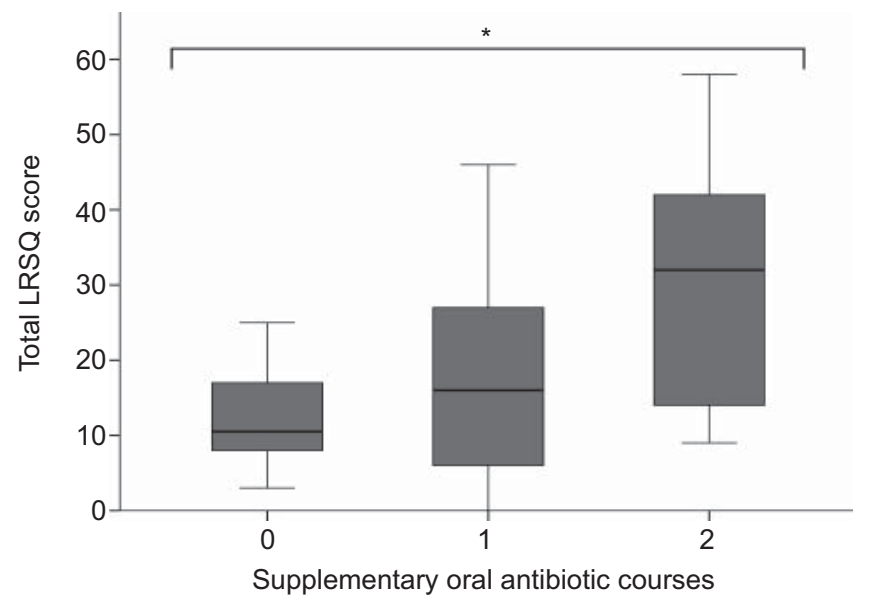

FIGURE 3. Total Liverpool Respiratory Symptom Questionnaire (LRSQ) scores for subjects treated with oral antibiotics. *: $p<0.05$.

\section{Discriminant validity: 6- to 12-yr-old age group}

"Stable" children with CF aged 6-12 yrs scored higher in total LRSQ and seven out of eight domain scores compared with controls (total score $14(5-33)$ versus $3(0-6) ; \mathrm{p}<0.001)$ (table 2 , fig. 1). Differences in total LRSQ score persisted even if only the nine children with $\mathrm{CF}$, entirely free of supplementary antibiotics for the study period, were included in the analysis $(\mathrm{p}<0.010)$.

For all children with CF (stable and unstable, $n=32$ ), correlations were examined between total LRSQ and current NCXR scores, Shwachman score and FEV1. Significant correlations were seen between total LRSQ score and FEV1 $(r=-0.45$, $\mathrm{p}<0.010 ; \mathrm{n}=32)$, FVC $(\mathrm{r}=-0.37, \mathrm{p}<0.050 ; \mathrm{n}=32)$ and Shwachman score $(\mathrm{r}=-0.58, \mathrm{p}<0.001 ; \mathrm{n}=31)$. The correlation between total score and NCXR score showed a positive trend, but did not reach statistical significance $(\mathrm{r}=0.311, \mathrm{p}=0.089 ; \mathrm{n}=31)$. Following classification of CF disease severity by FEV1, we compared total LRSQ scores across the "mild" and "moderate" groups with total scores for control patients (control patients did not complete spirometry). We noted a decline in

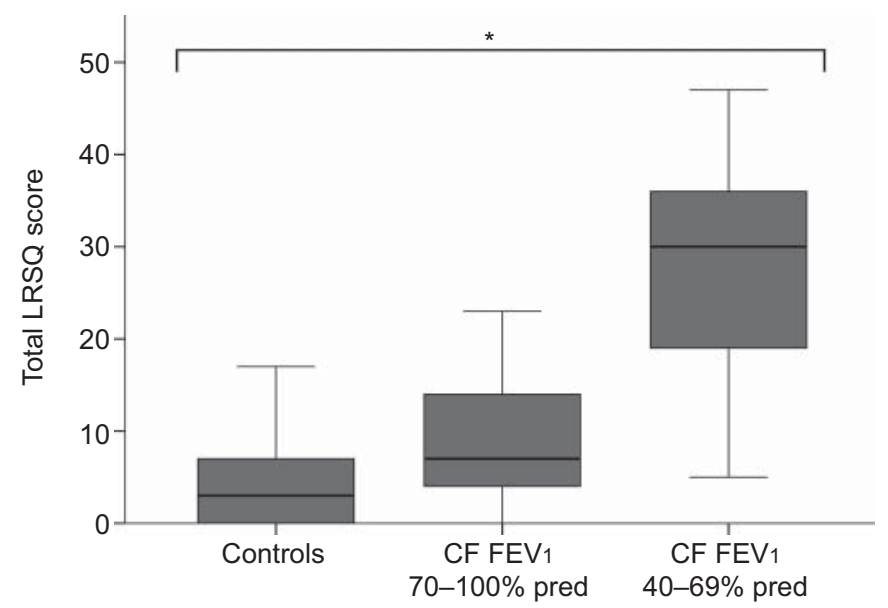

FIGURE 4. Total Liverpool Respiratory Symptom Questionnaire (LRSQ) scores for control subjects and cystic fibrosis (CF) subjects grouped by forced expiratory volume in $1 \mathrm{~s}\left(\mathrm{FEV}_{1}\right)$. \% pred: \% predicted. *: $\mathrm{p}<0.05$. median scores from those with moderate-to-mild disease severity to control patients $(\mathrm{p}<0.001)$ (fig. 4).

We compared LRSQ scores with P. aeruginosa carriage (fig. 5a). Total median scores were higher in those children who cultured $P$. aeruginosa at the time of LRSQ completion compared to those who were negative ( $P$. aeruginosa positive $23(16.8-34.5)(\mathrm{n}=12)$ versus $P$. aeruginosa negative 10 (3.5-24), $\mathrm{n}=18 ; \mathrm{p}<0.050)$. Pseudomonas carriage was classified according to the Leeds criteria. No statistically significant difference was found in scores across the groups, but a rising trend in LRSQ scores was seen (fig. 5b).

Finally, we analysed total LRSQ scores based on school attendance and found that median total LRSQ score was higher in those children with CF needing 1-2 days per month off school compared with those with full attendance (median 47 (29-64.5) versus 15 (3-23), $\mathrm{n}=5$ and 25, respectively; $\mathrm{p}<0.01$ ).

\section{Internal consistency}

In pre-school children with CF, the Cronbach's $\alpha$ coefficients in six out of eight domains ranged between 0.76-0.89, demonstrating acceptable-to-good internal consistency (table 3). The
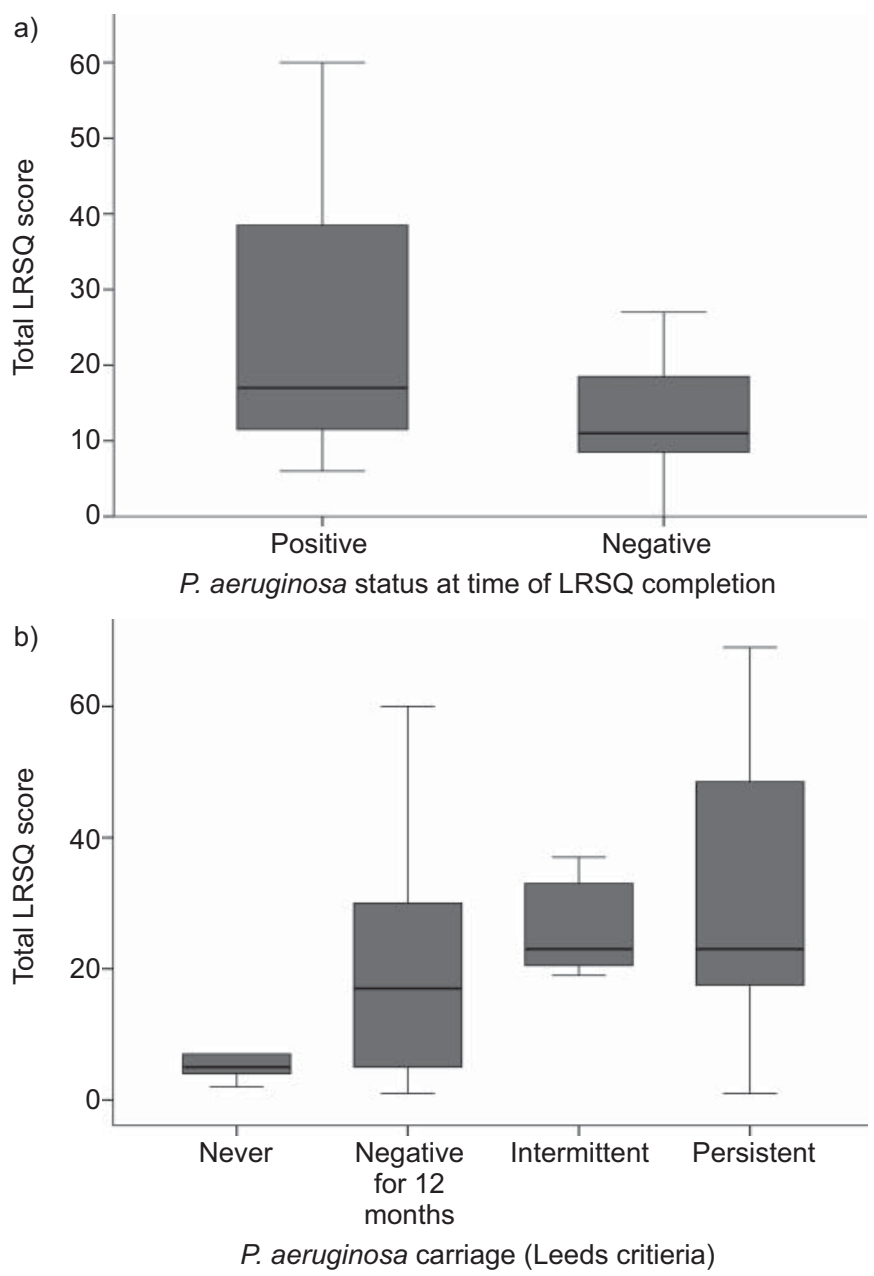

FIGURE 5. Total Liverpool Respiratory Symptom Questionnaire (LRSQ) scores according to a) Pseudomonas aeruginosa status at the time of LRSQ completion and b) $P$. aeruginosa carriage according to Leeds criteria. 


\begin{tabular}{|c|c|c|c|}
\hline \multirow{3}{*}{$\begin{array}{l}\text { TABLE } 3 \\
\text { Domain }\end{array}$} & \multicolumn{3}{|c|}{$\begin{array}{l}\text { Cronbach's } \alpha \text { coefficients for Liverpool } \\
\text { Respiratory Symptom Questionnaire domains }\end{array}$} \\
\hline & & \multicolumn{2}{|c|}{ Cystic fibrosis } \\
\hline & & $\leqslant 5$ yr-old age group & $6-12$-yrs-old age group \\
\hline \multicolumn{2}{|l|}{ Daytime } & 0.76 & 0.83 \\
\hline \multicolumn{2}{|l|}{ Night-time } & 0.64 & 0.83 \\
\hline \multicolumn{2}{|c|}{ Symptoms with colds } & 0.66 & 0.81 \\
\hline \multicolumn{2}{|c|}{ Interval symptoms } & 0.81 & 0.77 \\
\hline \multicolumn{2}{|c|}{ Activity symptoms } & 0.79 & 0.87 \\
\hline \multicolumn{2}{|c|}{ Other symptoms } & 0.80 & 0.75 \\
\hline \multicolumn{2}{|c|}{ Effects on child } & 0.89 & 0.76 \\
\hline \multicolumn{2}{|c|}{ Effects on family } & 0.79 & 0.74 \\
\hline
\end{tabular}

"night-time symptoms" domain had a coefficient of 0.64 , which improved to 0.71 if the question regarding snoring was removed. The "cold symptoms" domain had a coefficient of 0.66. Internal consistency was consistently acceptable-to-good across all domains in the $>5$-yr-old age group with CF (0.74$0.87)$.

\section{Ceiling and floor effects}

No ceiling or floor effects were observed in CF patient scores $(n=64)$. However, in pre-school control children, there were borderline floor effects in the total score $(19.6 \%)$. Floor effects were observed to a greater degree in the older control patients (40.5\% floor effect in total score). This further supports the suitability of the LRSQ to detecting respiratory symptoms in the CF population.

\section{DISCUSSION}

We have shown that the LRSQ total score successfully distinguishes "stable" CF patients from healthy children in the pre-school and in the 6- to 12-yr-old age group. These differences persisted when children with CF who had needed one course of oral "back-up" antibiotics in the previous 3 months were excluded. We have also shown that the LRSQ score correlates with school attendance and antibiotic usage over a 3-month period in children with CF. The LRSQ score therefore appears to distinguish children based on general clinical status within the CF cohort over a period of months. Correlations between LRSQ scores and other measures of respiratory status in the 6- to 12-yr-old age group highlight the potential role for this tool in both clinical and research paediatric CF settings. The LRSQ also allows respiratory symptom comparison across different paediatric diseases (previously validated in a non-CF pre-school group).

In the 6- to 12-yr-old CF group, children scored higher than controls in seven out of eight domains. Domain scores were generally higher in pre-school children with $\mathrm{CF}$, although statistical significance was only reached in two domains. In part, this reflects the higher scores seen in the healthy preschool children, which may be due to the frequency of viral respiratory infections seen in this age group. One of the domains where scores were higher in pre-school children with CF was "effect on the family". This is perhaps not surprising given that this domain covers how a child's respiratory symptoms impact on family life. More interestingly, scores for the "other respiratory symptom" domain were also higher than the control group. This domain covers noisy breathing not from the chest, fast breathing and noisy breathing from the back of the throat, all symptoms that may relate to increased large airway secretions.

To date, no formal validation of a respiratory symptom questionnaire for pre-school children with $\mathrm{CF}$ has been successful. In adults and adolescents, the CFQ-R and the Cystic Fibrosis Quality of Life Questionnaire (CFQoLQ) are widely used, reliable and valid. The CFQ-R has a fully validated child and parent completed version for children aged 6-13 yrs, but there is currently no fully validated preschool version [7-9, 15]. Although the CFQ-R respiratory domain has been validated and adopted as an outcome measure in clinical trials, its utility in paediatric clinical use (monitoring) may be limited by having only four items in the child version and six in the parent version [10, 19]. The LRSQ has potential advantages as it has been designed as an instrument for parental completion, is partially validated in those aged $\leqslant 5$ yrs and covers an extensive number of symptoms, whilst maintaining acceptability. Unlike other respiratory conditions, such as asthma [20], much of the focus in the development of respiratory symptom scores for CF has been on creating shorter term recall tools that are capable of identifying pulmonary exacerbations $[2,6]$. In contrast, the LRSQ is not designed to be used as a pulmonary exacerbation score. It assesses symptoms over a 3-month period and, as such, may have utility as a measure of longer term respiratory status (for example, incorporated into an annual review assessment). In addition, there is potential for the LRSQ to be used as a remote monitoring tool with parents being able to compare previous values and provide objective data for the $\mathrm{CF}$ team to remotely assess condition.

There has been debate within the literature surrounding the reliability of longer term symptom recall [21]. We have demonstrated that the LRSQ score can differentiate children based on number of antibiotic courses and school attendance over a 3-month period. This supports our assertion that the LRSQ is collecting data that reflects longer term symptomatology. There is also debate within the literature surrounding the reliability of symptom reporting in reflecting disease status. Many studies have examined the relationship between early inflammatory changes and other clinical parameters of disease (including symptomatology) in pre-school children. Some studies reported poor correlation between parent-reported symptoms and markers of infection and inflammation, lung function or computer tomography scan findings. However, these studies either recorded general symptoms without the use of an objective tool or used a tool that was not validated for use in young children with CF [22-25]. In this study, we have demonstrated a cross-sectional trend of increasing symptom development over pre-school years (in contrast to healthy controls), as well as correlation of symptom scores with other measures of both short- and longer-term clinical disease status. This suggests that the use of a standardised symptom questionnaire may yield more useful results. The correlation of LRSQ scores with other disease monitoring tools, which we know have limited sensitivity, does not confirm the questionnaire's value. The Shwachman and NCXR scores are 
known to be insensitive in young children, which may explain the lack of correlation in the pre-school group. Future studies investigating the correlation of LRSQ scores with early measures of structural, inflammatory or infective processes, such as bronchoalveolar lavage inflammatory markers and computed tomography changes, would be extremely useful, and would further assess the potential of this tool in identifying windows of opportunity for early intervention.

A source of bias in our study was the control group, with some recruits being children of healthcare staff. The higher incidence of passive household smoking in the CF group highlights the potential for socioeconomic status inequality between groups [26], but regression analysis did not demonstrate a confounding effect on LRSQ scores. It is of note that this study was conducted in an area with high levels of socioeconomic deprivation. $48 \%$ of CF patients were exposed to passive smoke within the home. This is highly concerning and highlights the need to enquire about passive smoking and offer smoking cessation support. Data about other possible confounding factors, including the age and sex of the person completing the questionnaire, nursery attendance and number of siblings (affecting exposure to respiratory pathogens), household pets and allergies were either not available or not assessed.

With regards to LRSQ validity, we have shown acceptable-togood internal consistency in six out of eight domains in preschool children and eight out of eight domains in older children. The "night-time symptoms" domain could be improved by excluding the question on snoring. The prevalence of snoring in children varies between 3.2 and $12 \%$ depending on age, and is primarily due to pharyngeal airway size. This physiological variation, the range of possible associated underlying pathologies, together with the potential bias of the arbitrary definition of snoring and reliance on parental reporting (who might not sleep in the same room), could render this item unreliable in assessing night-time respiratory symptoms within a paediatric population $[27,28]$. Previous studies have demonstrated moderate-to-good test-retest repeatability of the LRSQ, further indicating its validity [11]. The acceptability of the questionnaire to parents was also assessed previously and found to be practical and acceptable [11]. This is supported by the high percentage of correctly completed questionnaires (>99\%) in this study.

The wide range of total scores from CF patients provides reassurance that the questionnaire is capable of detecting differences, even within a stable CF paediatric population. Further evidence of discriminant validity is provided by the correlation of LRSQ scores with other outcome measures. Future studies will assess children at a time of instability and examine the scores for evidence of a ceiling effect. No floor effects were seen in total scores for CF patients, even at a time of stability. This was in contrast to healthy control patients in whom floor effects were seen. This indicates that the LRSQ is appropriate for detecting mild symptoms in a "stable" CF population.

Some other aspects of questionnaire validation were not assessed in this study, including inter-rater reliability and an assessment of the sensitivity of the LRSQ in detecting longitudinal changes in respiratory condition. Further limitations of the study include the relatively small numbers of patients within some of our subgroup analyses and the question of whether parental completion of a questionnaire is appropriate for older children. There is some conflict within the literature regarding concordance of child- and parent-reported respiratory symptoms [29, 30]. However, strong convergence of child/parent respiratory symptom reporting was confirmed in the CFQ-R child validation study (6- to 13-yr-old age group) [7]. This supports our use of parental assessment, which is particularly useful in younger children.

A limitation of this study is the absence of longitudinal data in individual patients. We are planning a large longitudinal study to more formally assess the sensitivity of the LRSQ to change in clinical status over time. This future study will also allow some aspects of psychometric validation, not possible in this study, to be assessed. We have shown that the LRSQ can distinguish "stable" children with CF from children with CF who are "unwell". The cross-sectional data presented highlights the potential sensitivity of this tool in detecting symptoms in preschool children. We believe this tantalising data highlights the potential of this measure for monitoring respiratory condition through childhood.

\section{Conclusions}

This study suggests that the LRSQ is a sensitive tool for detecting respiratory disease in the pre-school and school-age CF population. Correlations between LRSQ scores and other measures of CF disease severity in older children suggest that the questionnaire will have a role in the objective monitoring of long-term respiratory status. This may be a particularly important tool in pre-school children with $\mathrm{CF}$, where other clinical measures are limited.

\section{STATEMENT OF INTEREST}

None declared.

\section{ACKNOWLEDGEMENTS}

We would like to thank C. Darracott and G. Harrison (Respiratory Unit, Alder Hey Children's Hospital, Liverpool, UK) for collecting and recording the questionnaire data. We would like to acknowledge C. Powell (Cardiff University, Cardiff, UK) and N. Shaw (Respiratory Unit, Alder Hey Children's Hospital, Liverpool, UK) for their original work on the questionnaire. Acknowledgement also goes to K. Dwan (Dept of Biostatistics, University of Liverpool) for statistical support.

\section{REFERENCES}

1 Conway SP, Pond MN, Bowler I, et al. The chest radiograph in cystic-fibrosis - a new scoring system compared with the Chrispin-Norman and Brasfield Scores. Thorax 1994; 49: 860-862.

2 Mayer-Hamblett N, Ramsey BW, Kronmal RA. Advancing outcome measures for the new era of drug development in cystic fibrosis. Proc Am Thorac Soc 2007; 4: 370-377.

3 Zemanick ET, Harris JK, Conway S, et al. Measuring and improving respiratory outcomes in cystic fibrosis lung disease: opportunities and challenges to therapy. J Cyst Fibros 2010; 9: 1-16.

4 Quittner AL, Modi A, Cruz I. Systematic review of health-related quality of life measures for children with respiratory conditions. Paediatr Respir Rev 2008; 9: 220-232.

5 Cella D, Yount S, Rothrock N, et al. The Patient-Reported Outcomes Measurement Information System (PROMIS) Progress of an NIH roadmap cooperative group during its first two years. Med Care 2007; 45: Suppl. 5, S3-S11. 
6 Goss $\mathrm{CH}$, Edwards TC, Ramsey BW, et al. Patient-reported respiratory symptoms in cystic fibrosis. J Cyst Fibros 2009; 8: 245-252.

7 Modi AC, Quittner AL. Validation of a disease-specific measure of health-related quality of life for children with cystic fibrosis. J Pediatr Psychol 2003; 28: 535-545.

8 Gee L, Abbott J, Conway SP, et al. Development of a disease specific health related quality of life measure for adults and adolescents with cystic fibrosis. Thorax 2000; 55: 946-954.

9 Modi AC, Lim CS, Driscoll KA, et al. Changes in pediatric healthrelated quality of life in cystic fibrosis after iv antibiotic treatment for pulmonary exacerbations. J Clin Psychol Med Settings 2010; 17: 49-55.

10 Quittner AL, Modi AC, Wainwright C, et al. Determination of the minimal clinically important difference scores for the cystic fibrosis questionnaire - revised respiratory symptom scale in two populations of patients with cystic fibrosis and chronic Pseudomonas aeruginosa airway infection. Chest 2009; 135: 1610-1618.

11 Powell CVE, McNamara P, Solis A, et al. A parent completed questionnaire to describe the patterns of wheezing and other respiratory symptoms in infants and preschool children. Arch Dis Child 2002; 87: 376-379.

12 Luyt DK, Burton PR, Simpson H. Epidemiologic-study of wheeze, doctor diagnosed asthma, and cough in preschool-children in Leicestershire. BMJ 1993; 306: 1386-1390.

13 Hafen GM, Ranganathan SC, Robertson CF, et al. Clinical scoring systems in cystic fibrosis. Pediatr Pulmonol 2006; 41: 602-617.

14 Lee TWR, Brownlee KG, Conway SP, et al. Evaluation of a new definition for chronic Pseudomonas aeruginosa infection in cystic fibrosis patients. J Cyst Fibros 2003; 2: 29-34.

15 Quittner AL, Butt A, Messer MA, et al. Development and validation of the cystic fibrosis questionnaire in the United States - a health-related quality-of-life measure for cystic fibrosis. Chest 2005; 128: 2347-2354.

16 Nunnally J, Bernstein I, eds. Psychometric Theory. 3rd Edn. New York, McGraw-Hill, 1994.

17 Parent EC, Dang R, Hill D, et al. Score distribution of the scoliosis research society-22 questionnaire in subgroups of patients of all ages with idiopathic scoliosis. Spine 2010; 35: 568-577.
18 McHorney CA, Ware JE, Lu JFR, et al. The MOS 36 item short-form health survey (SF-36). 3. Tests of data quality, scaling assumptions, and reliability across diverse patient groups. Med Care 1994; 32: 40-66.

19 McCoy KS, Quittner AL, Oermann CM, et al. Inhaled aztreonam lysine for chronic airway Pseudomonas aeruginosa in cystic fibrosis. Am J Respir Crit Care Med 2008; 178: 921-928.

20 Asher MI, Keil U, Anderson HR, et al. International study of asthma and allergies in childhood (ISAAC) - rationale and methods. Eur Respir J 1995; 8: 483-491.

21 Stull DE, Leidy NK, Parasuraman B, et al. Optimal recall periods for patient-reported outcomes: challenges and potential solutions. Curr Med Res Opin 2009; 25: 929-942.

22 Stick SM, Brennan S, Murray C, et al. Bronchiectasis in infants and preschool children diagnosed with cystic fibrosis after newborn screening. J Pediatr, 155: 623-628.

23 Brennan S, Hall GL, Horak F, et al. Correlation of forced oscillation technique in preschool children with cystic fibrosis with pulmonary inflammation. Thorax 2005; 60: 159-163.

24 Dakin CJ, Numa AH, Wang H, et al. Inflammation, infection, and pulmonary function in infants and young children with cystic fibrosis. Am J Respir Crit Care Med 2002; 165: 904-910.

25 Armstrong DS, Hook SM, Jamsen KM, et al. Lower airway inflammation in infants with cystic fibrosis detected by newborn screening. Pediatr Pulmonol 2005; 40: 500-510.

26 Laaksonen $\mathrm{M}$, Rahkonen $\mathrm{O}$, Karvonen $\mathrm{S}$, et al. Socioeconomic status and smoking. Eur J Public Health 2005; 15: 262-269.

27 Corbo GM, Forastiere F, Agabiti N, et al. Snoring in 9- to 15-yearold children: risk factors and clinical relevance. Pediatrics 2001; 108: 1149-1154.

28 Corbo GM, Fuciarelli F, Foresi A, et al. Snoring in children association with respiratory symptoms and passive smoking. BMJ 1989; 299: 1491-1494.

29 Kieckhefer GM, Lentz MJ, Tsai S-Y, et al. Parent-child agreement in report of nighttime respiratory symptoms and sleep disruptions and quality. J Pediatr Health Care 2009; 23: 315-326.

30 Hoek G, Wypij D, Brunekreef B. Self-reporting versus parental reporting of acute respiratory symptoms of children and their relation to pulmonary function and air pollution. Int J Epidemiol 1999; 28: 293-299. 